\title{
Exploring solar-type pulsation with BRITE
}

\author{
T. Kallinger
}

Institut für Astronomie, Türkenschanzstrasse 17, 1180 Vienna, Austria

\section{Abstract}

It is evident that there is still no complete picture of how pulsation works in stars with convective envelopes. In the past few years the field of observing solartype oscillations has moved from ambiguous detections to firm measurements. However, I will demonstrate that BRITE is not an appropriate instrument to observe solar-type pulsation in sun-like stars, but rather perfectly suited to detect solar-type oscillations in stars cooler and more luminous than the Sun. Asteroseismology of red giants is still in its infancy and the few currently available high-precision data may not be adequate to answer the open questions. Using clever observing strategies, BRITE can help to significantly improve our understanding of the interaction between pulsation and convection, but also of stellar evolution during the important red giant phase in general.

\section{Introduction}

Our understanding of the Sun's structure has been revolutionised over the last three decades by helioseismology. This technique allows investigations of the solar interior by observing $p$-modes at the stellar surface.

Observing solar-type oscillations in other stars was for a long time not feasible due to the extremely small pulsation amplitudes. As high-precision radial velocity measurements from the ground and ultra-precise rapid photometry from space became available, detections of stochastically driven oscillations were reported for several sun-like stars. Examples include $\alpha$ Cen A (Bouchy \& Carrier 2002) and B (Kjeldsen et al. 2005) or Procyon A (Leccia et al. 2007 for radial velocity observations, Bruntt et al. 2005 for WIRE $^{1}$ photometry or Guenther et al. 2007 for MOST $^{2}$ photometry). Because of the very low amplitudes (some $\mathrm{ppm}$ in luminosity or some ten $\mathrm{cm} \cdot \mathrm{s}^{-1}$ in radial velocity, respectively), it is still an extremely challenging task to observe solar-type oscillations in G- or late F-type main-sequence (or slightly evolved) stars and the number of potential 


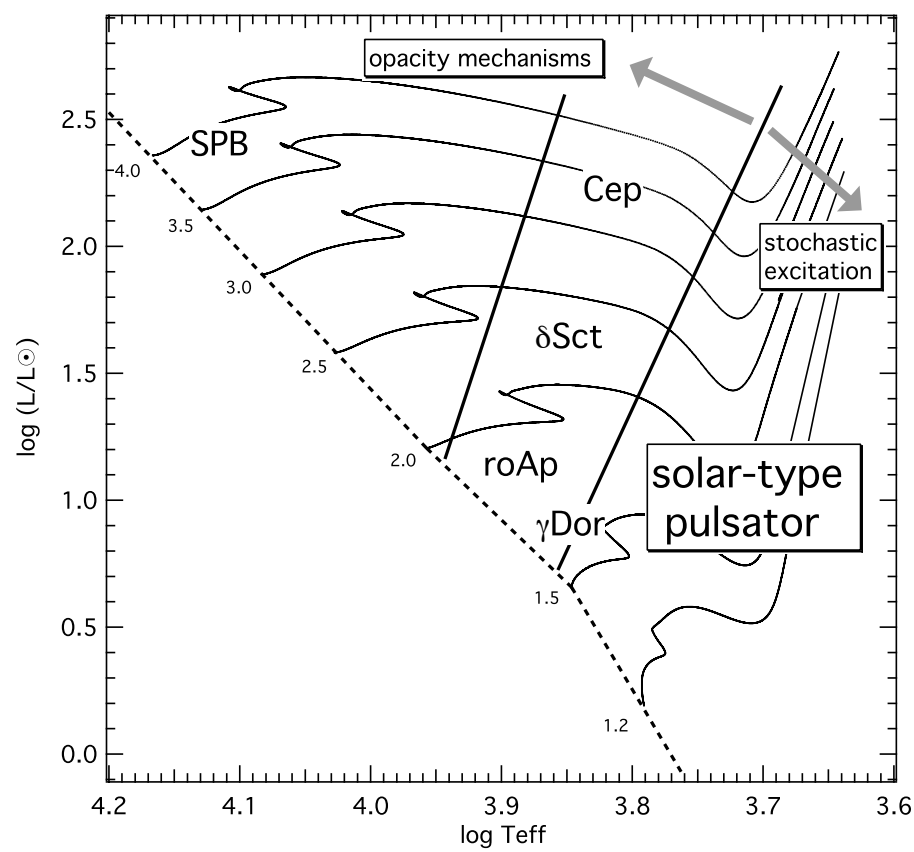

Figure 1: Theoretical HR-Diagram showing examples for regions where pulsating stars are observed.

targets in very limited.

For stars cooler and more luminous than the Sun, however, the amplitudes are greater (some hundred $\mathrm{ppm}$ or some $\mathrm{m} \cdot \mathrm{s}^{-1}$ ) and are more easily observable. The larger radii of red giants extend their pulsation periods from about 5 minutes in the Sun to a range of several hours to several days. This again complicates groundbased detections and frequency identifications, especially due to daily aliasing, making them perfect targets for space-based observations. Examples for pulsating red giants are $\epsilon$ Oph (De Ridder et al. 2006 for radial velocity observations and Kallinger et al. 2007 for MOST photometry) or $\xi$ Hya (Frandsen et al. 2002). Currently about a dozen of red giants showing solartype pulsation are known and at least three additional candidates have been discovered by MOST (not yet published).

\footnotetext{
${ }^{1}$ Wide-field InfraRed Explorer star tracker photometry

${ }^{2}$ Microvariability and Oscillation in STars; a Canadian micro-satellite mission with assistance from the University of Vienna, Austria
} 


\section{Solar-type pulsation}

Looking at the HR-Diagram (Fig. 1), one can find various regions where stars tend to show variability. Intermediate mass stars $(>\sim 1.5 \mathrm{M} \odot)$ cross the classical $\delta$ Scuti instability strip (in the center of the HR-Diagram) during their evolution from the main sequence towards the subgiant branch. During this evolutionary phase, stars have an appropriate internal configuration to become pulsationally unstable, presumably driven by the $\kappa$ mechanism in the hydrogen and helium ionization zones.

Beyond the red edge of the instability strip, stars possess convective envelopes and can show mode instability due to perturbations of their convective flux. It is believed that the acoustic noise generated by the convection in the star's resonant cavity may drive intrinsically stable (hence damped) $p$-mode pulsation, resulting in damped and stochastically excited (solar-type) pulsation. Unlike $p$-modes in stars in the classical instability strip, which can coherently oscillate for millions of years, acoustic modes caused by turbulence in the convective envelopes have much shorter lifetimes. Pulsation of this nature can be characterized, in its simplest description, as a superposition of stochastically excited and intrinsically damped harmonic oscillations. The Fourier transform of a set of such incoherent signals produces a broadened peak in the power spectrum, a so-called Lorentzian profile, provided that the time series is infinitely long. For finite data sets, the Fourier transform enforces artificial beating frequencies, randomly distributed under the Lorentzian profile, to reproduce the amplitude (and phase) modulation of the signal.

Such a damped and re-excited mode carries, in addition to the information about the stellar interior (via its frequency), information about the convection zone via its peak envelope in the power spectrum. This can be used to test and improve convection theories as well as nonadiabatic pulsation theory. Fig. 2 (left panel) displays the noise-free synthetic time series of a mono-periodic but strongly damped (with a damping rate of 0.1 per day) and randomly re-excited signal as well as the Fourier amplitude spectrum of the same signal (right panel). The intrinsic mode lifetime (the inverse damping rate) is determined from the line width $\Gamma$ of a Lorentzian profile function (grey-shaded area in Fig.2) fitted to the amplitude spectrum with $\Gamma=1 /(\pi \tau)$ where $\tau$ denotes the mode lifetime.

To date, direct measurements of mode lifetimes (via mode line widths) are available only for the Sun. However, there is the possibility to estimate mode lifetimes indirectly. For solar-type oscillation, the scatter of the largest amplitude peak under the Lorentzian profile around the real mode frequency (center of the Lorentzian profile) is a function of the intrinsic mode lifetime (and the data set length as well as signal-to-noise ratio of the mode). By comparing the observed frequency scatter with extensive simulations, Kjeldsen 

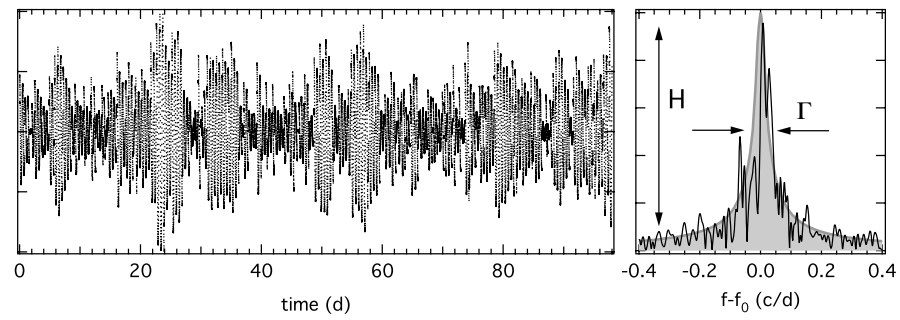

Figure 2: Synthetic light curve of a mono-periodic but strongly damped and stochastically re-excited signal. The Fourier transform enforces artificial beat frequencies, to reproduce the amplitude (and phase) modulation of the signal, causing randomly distributed peaks under a Lorentzian profile (grey-shaded area). The width and height of the profile depend on the damping rate (inverse lifetime).

et al. (2005) determined the mode lifetimes of $\alpha$ Cen A and B to be roughly 2 and 3 days, respectively. Using a similar approach, Kallinger et al. (2007) found a mode lifetime of about 10 to 20 days for $p$-modes in $\epsilon$ Oph.

\section{Solar-type pulsation in sun-like stars}

As already mentioned, solar-type oscillations in $G$ and late F-type stars from the main sequence to the end of the hydrogen core-burning (which are generally referred to as sun-like stars) show very small amplitudes and the number of sunlike stars where solar-type oscillations have been detected is quite limited. To date, all known solar-type pulsators have been discovered using high-pression radial velocity measurements from ground. To observe them photometrically, space instruments are needed. Unfortunately, the currently operating photometric satellite missions MOST and WIRE are not sensitive enough to unambiguously observe solar-type oscillations in sun-like stars, even the brightest ones (except for $\alpha$ Cen A). In other words, the photometric amplitudes are smaller than expected (e.g., one of the main goals of MOST was to detect solar-type oscillations in sun-like stars - and the detection limit is actually better than the requirements). The french-lead mission $\mathrm{COROT}^{3}$ should be capable to fill this lack of photometric observations.

However, some sun-like stars are bright enough to be observable with BRITE. E.g., Fig 3 shows a Fourier power spectrum of a simulated photometric time series as we would expect it for the F5 IV star Procyon A. Mode frequencies and amplitudes (scaled from radial velocity amplitudes) are taken from Leccia

\footnotetext{
${ }^{3}$ COnvection, ROtation and planetary Transits
} 


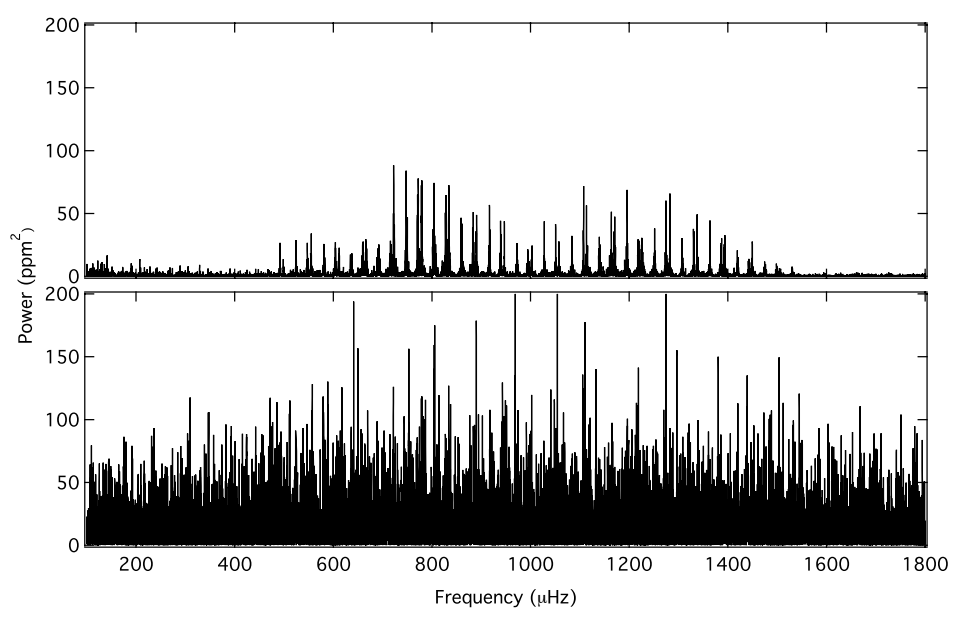

Figure 3: Top panel: Fourier power spectrum of a synthetic photometric time series based on radial velocity observations of Procyon A. Bottom panel: Same as above but as expected for typical BRITE observations (300ppm point-to-point scatter and $30 \%$ duty cycle).

et al. (2007) and have a lifetime of 2 days. The low frequency power comes from a simple granulation noise model with parameters estimated from Bruntt et al. (2005). The power spectrum given in the bottom panel of Fig.3 should illustrate what we can typically expect when observing Procyon A with BRITE. A typical point-to-point scatter of $300 \mathrm{ppm}$ (for a $\mathrm{V}=0.3 \mathrm{mag}$ star) was added (A. Kaiser, private comm.), the time series was resampled to $6 \mathrm{sec}$ exposures and the duty cycle was restricted to $30 \%$ (typical for a single BRITE satellite). It is obvious that the $p$-mode signal gets lost in the noise and instrumental signal. Even when increasing the duty cycle to $90 \%$ and decreasing the pointto-point-scatter by $50 \%$, no clear signature of the $p$-mode pattern is evident. Hence, I strongly recommend not to attempt to observe solar-type oscillations in sun-like stars with BRITE.

\section{Solar-type pulsation on the giant branch}

The situation changes for more developed stars. As the amplitudes of solartype oscillation scale with stellar luminosity the signal is more easily observable in red giants. E.g., a typical $\mathrm{K} 0$ giant has a luminosity of $50 \mathrm{~L} \odot$ and one can expect an amplitude of about 200ppm for solar-type oscillation (compared to about $4 \mathrm{ppm}$ for the Sun) for such a star. However, the increased stellar radius 
extends the pulsation periods from about 5 minutes in the Sun to a range of some hours to days. This complicates ground based observations, especially due to daily aliasing, and calls for space observations. Besides the absence of the Earth's atmosphere, space based observations of pulsating cool giants benefit from the satellite orbit. All photometric space missions so far fly in Low Earth Orbits with an orbital period on the order of 100 minutes. Hence, uninterrupted observations with orbital gaps (as it is planed for BRITE) yield a clean spectral window function in the low frequency domain (below $\sim 10 \mathrm{c} / \mathrm{d}$ ) but with alias peaks at roughly $14 \mathrm{c} / \mathrm{d}$ (and multiples thereof). This is of particular interest when observing solar-type oscillation in red giant stars because the frequency regime of pulsation is well below the orbital frequency and instrumental aliases do not distort the intrinsic eigenspectrum.

It has been suggested by several authors that only radial $p$-modes should be observable in red giants while nonradial modes are assumed to be damped. The theoretical position is unclear. Dziembowski et al. (2001) showed the existence of strongly trapped unstable nonradial modes in the central regions of giants in between very closely spaced modes of the same degree $l$. These modes follow the same pattern of frequency spacing as radial acoustic waves and correspond to mixed $g$ - and $p$-modes. Only in their linear stability analysis, which includes the effects of turbulent pressure based on the mixing-length formalism, they find amplitudes of the radial modes of the sub-giant star $\alpha \mathrm{UMa}$ to be much smaller than observed, with the nonradial mode amplitudes predicted to be even smaller. On the observational side, Hekker et al. (2006) note that the line profile variations of several pulsating red giants suggest the existence of nonradial modes. Apart from this, Stello et al. (2006) found evidence that the mode lifetimes of oscillations in the $\mathrm{G} 7$ giant $\xi$ Hya are only about 2 days which is roughly 5 to 10 times shorter than what is inferred from theoretical considerations (Houdek \& Gough 2002).

Most recently, Kallinger et al. (2007) found clear evidence for radial and nonradial modes in the $\mathrm{G} 9.5$ giant $\epsilon \mathrm{Oph}$ in both luminosity and radial velocity measurements based on MOST observations and spectroscopic time series (De Ridder et al. 2006), respectively. They fit 18 of the 21 most significant photometric frequencies to radial and $l=1,2$ and 3 modes (see left panel of Fig.4). The best fitting model lies within the uncertainty of the known position in the HR-Diagram and its interferometrically determined radius (see right panel of Fig.4). The observed frequency scatter is consistent with mode lifetimes of 10 to 20 days. 

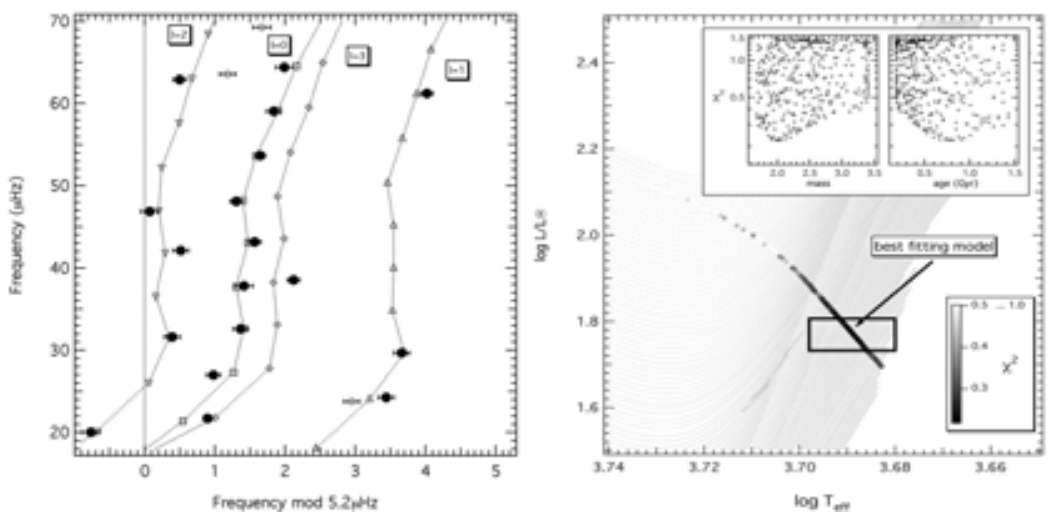

Figure 4: Left panel: Echelle diagram of the 21 most significant photometric modes of $\epsilon$ Oph used to constrain the best fit. Filled dots corresponds to the 18 frequencies matching model frequencies indicated by grey symbols and connected by line segments. Right panel: Theoretical HR-Diagram showing the uncertainty box location of $\epsilon$ Oph and a subset of the stellar model grid used for pulsation analysis (small grey dots). The grey scale (large dots) gives the $\chi^{2}$ values derived from the differences between observed and model modes where the scale is limited to values smaller than 1 . The location of the best fitting model is indicated by the arrow. The inserts illustrate $\chi^{2}$ as a function of model mass and age, respectively.

\section{Observing strategies}

As outlined in the previous section, pulsating $\mathrm{G}$ and $\mathrm{K}$ giants are perfect candidates for photometric long-term observations with BRITE. Contrary to sun-like stars, solar-type oscillations in red giants exhibit amplitudes large enough to be clearly detectable with BRITE. The frequency regime of pulsation fits into a frequency range free from instrumental distortion. Additionally, there are many potential targets with well known fundamental parameters (even the radii are well constrained for most bright giant stars) bright enough to meet the mission requirements.

In terms of a reasonable target selection, however, it has to be mentioned that more luminous (hence larger amplitudes and in most cases also brighter) does not necessarily mean "better" targets. The acoustic cut-off frequency as well as the large frequency separation are inversely proportional to the stellar radius. In other words, for more luminous stars (with a larger radius), the frequency range of pulsation becomes narrower and moves to lower frequencies. This leads to the fact that at a certain point the Lorentzian profiles of consecutive modes start to overlap, making the identification of individual modes 

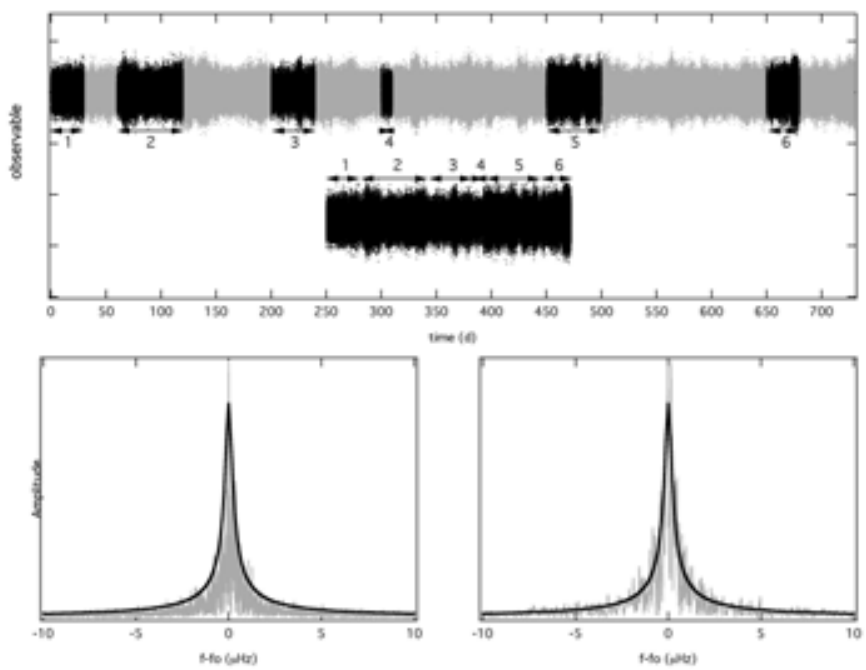

Figure 5: Synthetic time series including a stochastically excited damped signal illustrating that different observations of arbitrary length and epoch stitched together yield a reasonable result. The bottom left panel shows the Fourier power spectrum of the entire data set (including a Lorentzian fit). The bottom right panel gives the same but only for the black parts of the simulated time series stitched together.

nearly impossible. Hence, I propose to focus on targets filling up the undisturbed frequency range (up to about $10 \mathrm{c} / \mathrm{d}$ ) and having a large (but also small) frequency separation large enough to obtain clearly separated Lorentzian profiles. This sets a limit to stellar radii roughly smaller than $15 \mathrm{R} \odot$ for intermediate mass stars assuming lifetimes longer than 10 days.

To date, only relatively short data sets (on the order of some weeks) of pulsating red giants are available where the fact that the Fourier peak width due to the data set length is comparable to the width of the Lorentzian profile prevents a direct determination of the mode lifetimes (via the profile width). Hence, the current and future challenge in observing solar-type oscillation in red giants is to obtain data sets long enough to actually "see" the Lorentzian profiles in the power spectrum. Therefore, data sets 5 to 10 times as long as the mode lifetime are necessary, corresponding to more than 100 days of continuous observations. Entering a new era of red giant observations, COROT will observe several dozens of red giants for up to 150 days in the Exoplanet program. Unfortunately, only faint stars in small areas in the sky with poorly or even unknown fundamental parameters can be observed, which complicates detailed asteroseismic investigations. For the near future BRITE will be the 
only instrument capable to observe bright red giants for more than some weeks from space.

Finally, I want to mention that observing red giants for a long time does not necessarily mean to observe them continuously. A damped and stochastically excited signal has the attribute that is not coherent. Hence it has no "phase" in a classical sense like a normal sinusoidal signal. This has the advantage that a solar-type pulsator can be observed during several time-slots and the subsets can be stitched together without paying attention to the gaps (keeping the gaps degrades the spectral window functions). Fig. 5 illustrates such an observing strategy and shows that no artificial signal distorts the power spectrum of a "pseudo-continiuous" time series.

Acknowledgments. Financial support was received from the Austrian Research Promotion Agency (FFG) and the Austrian Science Fund (FWF P17580)

\section{References}

Bouchy ,F., Carrier, F. 2002, A\&A, 390, 205

Bruntt, H., Kjeldsen, H., Buzasi, D. L., et al. 2005, ApJ, 633, 440

De Ridder J., Barban C., Carrier F., et al. 2006, A\&A, 448, 689

Dziembowski, W. A., Gough, D. O., Houdek, G., et al. 2001, MNRAS, 328, 601

Frandsen, S., Carrier, F., Aerts, C., et al. 2002, A\&A, 394, L5

Guenther, D. B., Kallinger, T., Reegen, P., et al. 2007, CoAst, 151, 5

Hekker, S., Aerts, C., De Ridder, J., Carrier, F. 2006, A\&A, 458, 931

Houdek, G., Gough, D. O. 2002, MNRAS, 336, L65

Kallinger, T., Guenther, D. B., Matthews, J. M., et al. 2007, A\&A, in press

Kjeldsen, H., Bedding, T. R., Butler, P., et al. 2005, ApJ, 635, 1281

Leccia, S., Kjeldsen, H., Bonanno, A., et al. 2007, A\&A, 464, 1059

Stello, D., Kjeldsen, H., Bedding, T. R., Buzasi, D. 2006, A\&A, 448, 709 